\title{
Médiévales
}

Langues, Textes, Histoire

59 | automne 2010

Théâtres du Moyen Âge

\section{Le théâtre dans la ville : pour une histoire sociale des représentations dramatiques}

Drama in the City : toward a Social History of Theatrical Performances

\section{Matthieu Bonicel et Katell Lavéant}

\section{(2) OpenEdition}

1 Journals

Édition électronique

URL : https://journals.openedition.org/medievales/6088

DOI : 10.4000/medievales.6088

ISSN : 1777-5892

Éditeur

Presses universitaires de Vincennes

Édition imprimée

Date de publication : 31 décembre 2010

Pagination : 91-105

ISBN : 978-2-84292-267-2

ISSN : 0751-2708

Référence électronique

Matthieu Bonicel et Katell Lavéant, «Le théâtre dans la ville : pour une histoire sociale des

représentations dramatiques », Médiévales [En ligne], 59 | automne 2010, mis en ligne le 20 mars 2013 consulté le 24 avril 2022. URL : http://journals.openedition.org/medievales/6088 ; DOI : https:// doi.org/10.4000/medievales.6088 
Médiévales 59, automne 2010, p. 91-105

Matthieu BONICEL

Katell LAVÉANT

\author{
LE THÉÂTRE DANS LA VILLE: \\ POUR UNE HISTOIRE SOCIALE \\ DES REPRÉSENTATIONS DRAMATIQUES
}

Parmi les formules visant à décrire le théâtre de la fin du Moyen Âge contre lesquelles les historiens spécialistes de ce domaine sont amenés à se positionner, une en particulier hante les manuels scolaires: l'essence du théâtre médiéval aurait résidé dans deux buts, l'édification (par les mystères et moralités) et le divertissement (par les farces et sotties) du public médiéval. Cette observation, que l'on pourrait penser frappée au coin du bon sens, présuppose cependant une vision du phénomène dramatique réduite à la communication exclusive entre acteurs et spectateurs, ou plutôt des acteurs au public (ce dernier apparaissant de fait comme assistant passivement à la représentation), en laissant de côté la perception de l'activité dramatique dans son contexte social. Ainsi, elle réduit le théâtre à la représentation et à sa dimension spectaculaire, en effaçant l'importance du phénomène performatif pour la société médiévale dans son ensemble et en passant à côté de son importance économique, symbolique et politique ${ }^{1}$.

Or nous sommes convaincus que, pour évaluer à leur juste mesure la place et l'influence du théâtre comme phénomène social à la fin du Moyen Âge, il nous faut opérer un retour aux sources historiques, souvent convoquées pour documenter la représentation d'un texte de théâtre, mais encore trop rarement

1. Pour désigner le phénomène théâtral, nous utiliserons ici plusieurs termes proches, mais avec des sens légèrement différents, comme suit: la «représentation» désigne l'espace-temps dans lequel l'activité dramatique mise en scène est proposée aux spectateurs; le «spectacle» renvoie au dispositif global dans son organisation pratique et matérielle; la «performance» couvre un champ plus large, celui par lequel le phénomène théâtral est saisi dans le lien qui est créé avec les spectateurs, c'est-à-dire en prenant en compte non plus seulement ce qui est représenté, mais également son impact sur un public et dans un contexte socio-politique spécifique. 
étudiées pour cerner les cultures dramatiques. Le problème qui se pose alors est simple mais appelle plusieurs réponses, qui ne s'excluent pas, mais se complètent: quelles sources sont disponibles pour contribuer à l'histoire du théâtre, et comment les appréhender?

Nous voulons ici proposer quelques pistes de réflexion à partir de nos propres recherches, pour souligner à quels problèmes et à quels enjeux méthodologiques est confronté l'historien du théâtre, à travers une sélection d'exemples tirés de nos espaces d'étude respectifs - Avignon (M. Bonicel) et l'espace Flandres-Hainaut-Picardie (K. Lavéant) ${ }^{2}$. L'étude du premier de ces espaces permettra de mettre en évidence la nécessité d'opérer un retour aux sources, et montrera l'intérêt de centrer une telle étude sur les fonds comptables. L'approche de la deuxième région géographique nous donnera ensuite la possibilité de confirmer l'utilité d'une telle approche pour renouveler l'étude de questions complexes, en reprenant à titre d'exemple celle, essentielle, du (ou des) public(s) du théâtre médiéval.

\section{Produire la performance : l'étude ciblée d'une aire géographique}

La recherche de sources historiques concernant le théâtre médiéval dans un espace géographique cohérent d'un point de vue politique ou social conduit souvent le chercheur à s'interroger sur le contexte documentaire dans lequel il se trouve et à tenter d'identifier les producteurs de documents pouvant l'intéresser. Cette considération semble évidente mais elle se révèle d'une particulière vérité avec un sujet aussi «thématique » que le théâtre médiéval, pour lequel on trouve au final, par rapport à la masse des autres sujets, assez peu de documents. Une appréhension globale du producteur dans son ensemble est de notre point de vue nécessaire car elle peut conduire à identifier comme relevant du domaine spectaculaire, ou du moins performatif, un certain nombre de manifestations. Cette identification repose avant tout sur une étude des sources, de leur forme documentaire, de leur circuit d'enregistrement, et des personnes qui sont impliquées dans leur production. Ce type d'étude nous conduit au final à identifier le contexte documentaire de production de sources intéressant l'histoire du spectaculaire ou du performatif, ce qui permet in fine d'acquérir un indispensable flair archivistique, qui économise par la suite au chercheur des dépouillements longs, fastidieux et potentiellement stériles.

2. M. Bonicel, Arts et gens du spectacle à Avignon à la fin du Moyen Âge, Thèse pour le diplôme d'archiviste paléographe, École nationale des chartes, Paris, 2006; K. LAVÉANT, Théâtre et culture dramatique dans les villes des Pays-Bas méridionaux, $X V^{e}$-XVI $e^{e}$ siècles, Thèse de doctorat, université d'Amsterdam, 2007, à paraître aux éditions Paradigmes, Orléans, sous le titre Un théâtre des frontières. La culture dramatique dans les provinces du Nord (XVe-XVI siècles). 
En effet, lorsque l'on s'attache à un fonds d'archives où nombre de séries sont conservées pratiquement en continu, comme c'est le cas à Avignon pour la période 1450-1550, le recoupement des informations déjà en notre possession au départ de l'étude, issues par exemple du dépouillement des articles des revues locales, n'a qu'un temps ${ }^{3}$. Ce travail permet certes de se familiariser avec l'organisation de la documentation, mais arrive un moment où il faut considérer l'institution productrice dans son ensemble, et identifier aussi précisément que possible un certain nombre de critères pouvant être croisés afin de faciliter les dépouillements sériels. En outre, la proximité des sources conservées permet de constater qu'un grand nombre de manifestations publiques, telles que les funérailles, réelles ou fictives, à Avignon (par exemple pour la mort d'un roi de France), offre la possibilité de les rapprocher, sinon du théâtre à proprement parler, au moins du phénomène performatif. En effet, ces événements font appel aux mêmes fournisseurs (peintres, tailleurs, menuisiers par exemple), pour le même type de produits que pour l'organisation de représentations de mystères et autres pièces subventionnées par la municipalité. Ils mobilisent des volumes financiers comparables et comportent une forme de mise en espace qui apparaît dans la comptabilité par exemple lorsque l'on détaille le volume des cierges en fonction de leur positionnement devant tel ou tel dignitaire dans le chœur de l'église, comme pour la messe célébrée à Avignon lors de la mort du pape Pie IV en 1484.

\section{Les sources comptables}

Nous ne pouvons malheureusement pas donner ici une description complète du fonctionnement des différentes sources municipales à la fin du Moyen Âge, ce qui est cependant indispensable à toute étude des performances à travers cette documentation. On aurait ainsi intérêt à étudier les documents narratifs s'apparentant à des chroniques (pour Avignon, un ensemble intitulé «correspondance des consuls», série AA; pour les villes du Nord, les récits d'entrées joyeuses conservés dans les archives municipales et parfois également imprimés). Ce complément par rapport aux sources comptables est évidemment fort utile, notamment pour avoir la chronologie précise des événements et la description de leur déroulement, difficilement percep-

3. Soulignons cependant l'intérêt de consulter ces articles qui, bien qu'anciens le plus souvent, constituent toujours un point de départ essentiel pour de nouvelles recherches sur une région spécifique. Pour Avignon, on peut citer: P. PANSIER, «Les débuts du théâtre à Avignon à la fin du $\mathrm{XV}^{\mathrm{e}}$ siècle», Annales d'Avignon et du Comtat venaissin, VI, 1919, p. 5-52, ou L. Duhamel, «Le passage de César Borgia à Avignon », Bulletin du CTHS, 1889, p. 103-106. Plus globalement, sur les premiers historiens du théâtre, nous conseillons la lecture de ce recueil à paraître: M. BouHAÏKGironès, V. Dominguez et J. Koopmans éds., Les Pères du théâtre médiéval. Examen critique de la constitution d'un savoir académique. 
tibles dans la comptabilité. En revanche, toutes les représentations n'y sont pas décrites, ces documents n'étant pas du tout systématiques. Les délibérations du Conseil de ville, dans la série BB, peuvent aussi être un bon moyen de repérer les spectacles et d'en identifier le promoteur, voire d'étudier un système d'autorisation des représentations (ce type de document permet notamment de retracer le parcours de compagnies d'acteurs itinérantes dans l'espace Flandres-Hainaut-Picardie). Pour une analyse plus précise de ces sources, nous renvoyons le lecteur à nos travaux ${ }^{4}$.

Les documents comptables constituent bien souvent l'ensemble le plus exploitable d'un point de vue sériel, et le plus fiable pour rassembler des informations factuelles. En effet, lorsque l'on a la chance de conserver des séries de comptes continues il est possible d'envisager un repérage, non pas systématique, mais au moins séquentiel, d'un assez grand nombre de manifestations à caractère performatif. Il est en revanche indispensable de bien préciser le contexte de production auquel on s'attache et d'identifier clairement un producteur unique, afin d'exploiter des éléments bel et bien comparables. Notre étude portera ici sur la municipalité d'Avignon en tant que producteur de spectacles, en laissant de côté les institutions ecclésiastiques, pourtant nombreuses dans la cité.

La comptabilité d'une ville comme Avignon présente le grand avantage d'être balisée par trois grandes catégories de documents, au sein de la série CC du cadre de classement ${ }^{5}$, qui permettent des recoupements, voire des comblements d'information lorsqu'apparaît un «trou» dans l'une ou l'autre série: le registre du trésorier général, le registre des mandats, et les mandats, ou pièces à l'appui. Le premier document, tenu par le trésorier général lui-même et renouvelé chaque année avec l'élection d'un nouveau trésorier, se limite en général à donner le nom du bénéficiaire d'un paiement et le montant de ce dernier. Il est ainsi possible, afin de rendre plus rapide un dépouillement systématique de ces registres, de sonder plus particulièrement les paiements aux courriers de la ville (en dehors de leur salaire, payé tous les mois et dont le montant fixe est facilement repérable) ${ }^{6}$. Certaines fêtes, comme Carême Entrant, donnant régulière-

4. Voir note 1.

5. Pour une description détaillée du fonds des archives communales d'Avignon, on peut se reporter au précieux instrument de recherche de F. CHAUZAT et C.-F. Hollard, Répertoire numérique des archives communales d'Avignon antérieures à 1790 déposées aux Archives départementales, Avignon, 1995.

6. On peut en effet identifier clairement les courriers de la ville, et avant eux les maîtres des rues, comme les principaux employés municipaux délégués à l'organisation des performances. Ils se voient confier, souvent de manière globale, l'organisation des entrées, banquets, festivités publiques, et perçoivent la plupart du temps la totalité des subsides municipaux, à charge pour eux de les redistribuer entre les différents intervenants. Cette constatation faite, il est ensuite facile de repérer rapidement, et de manière séquentielle, les paiements effectués aux courriers de la ville dans les 
ment lieu à des banquets avec spectacle, il peut être intéressant de calculer cette date d'une année sur l'autre pour être particulièrement attentif aux paiements importants réalisés durant cette période.

Le registre donnant le numéro de mandat qui ordonne le paiement, il est aisé, une fois un mouvement comptable intéressant repéré, d'aller vérifier dans les liasses de mandats. Ces dernières sont constituées de pièces individuelles comprenant un ordre de paiement en latin de la main du notaire et secrétaire de la ville, et d'une liste détaillée, parfois rédigée par le créancier (ou un intermédiaire chargé d'écrire pour lui). Pour une manifestation importante, comme l'entrée de César Borgia à Avignon en 1498, le seul mandat décrivant les spectacles de théâtre organisés le long du passage du cortège est constitué d'un cahier de plusieurs pages décrivant précisément tous les achats nécessaires (matériaux de construction, textile, cierges), les paiements des artistes, et la destination précise de ce paiement. La description va donc bien au-delà d'une simple liste de fournitures, elle permet précisément de reconstituer le parcours dramatique de l'événement et d'en estimer le coût total.

Le registre des mandats, enfin, comprend une copie du texte des mandats, réalisée par le notaire et secrétaire de la ville lorsque celui-ci récupère le mandat après paiement par le trésorier. Il s'agit souvent d'un volume très épais tenu sur plusieurs années. Outil de contrôle à l'origine, il est aisé d'y avoir recours lorsqu'il existe un manque dans les liasses de mandats.

\section{Intérêt des sources comptables : la matérialité des représentations}

L'intérêt évident des sources comptables pour l'étude du théâtre médiéval est qu'elles nous apportent des éléments précis pour l'étude matérielle des représentations. L'étude de pièces comptables dans un contexte déterminé nous permet, d'une part, d'estimer le coût des représentations, et, d'autre part, d'en estimer la portée à l'échelle plus globale de l'économie d'une collectivité.

Les pièces à l'appui d'une comptabilité municipale nous donnent beaucoup de renseignements précieux pour percevoir la matérialité des spectacles à la fin du Moyen Âge. Elles indiquent souvent la liste précise de tous les éléments ayant été achetés pour former le décor, les costumes, l'aménagement des espaces publics, le salaire des intervenants. Il est aussi possible d'y percevoir les éléments les plus coûteux. Le textile et l'éclairage sont parmi les postes les plus importants, à tel point que le drap est parfois simplement loué (notamment pour les grands pans de tissus destinés par exemple à couvrir des parties de bâtiments), de même que les cierges que l'on pèse chez le fournisseur avant et 
après utilisation et pour lesquels on ne paie que la cire effectivement consommée. Si on reprend l'exemple de l'entrée de César Borgia en 1498 à Avignon, le coût total des festivités s'élève à 1905 florins. Les principaux postes sont le textile $(49 \%)$, les frais de bouche $(23 \%)$, les frais artistiques purs (théâtre, danse, musique, $9 \%$ ), la construction des infrastructures $(7 \%)$, les frais de décors et de peinture (6\%), les salaires autres que ceux des artistes $(6 \%)$.

En ce qui concerne le théâtre à proprement parler, il est assez fréquent qu'un personnage se voie remettre une somme d'argent globale pour la composition et l'exécution d'un jeu ou d'une farce. Cette somme est alors visiblement destinée à couvrir ses frais d'écriture ou de mise en scène et le salaire de ses comédiens. Certains citoyens ont parfois la délégation de la ville pour organiser eux-mêmes l'ensemble des spectacles autour d'un point précis, par exemple lors du passage d'une grande entrée. Ainsi, en 1498, à Avignon, Pierre de Sarrachana est mandaté pour prendre en charge l'échafaud de la Porte Saint-Lazare. Il remet par la suite un compte précis de cet événement au notaire et secrétaire de la ville pour obtenir remboursement. Un autre citoyen, Jean Lorin, se voit confier l'organisation des danses à l'hôtel de ville pour cette même entrée.

\section{Les modes de financement}

La majeure partie des subsides de la ville d'Avignon provient aux $\mathrm{Xv}^{\mathrm{e}}$ et $\mathrm{XVI}^{\mathrm{e}}$ siècles des impôts indirects, ou gabelles, essentiellement prélevés sur les marchandises entrant et sortant de la cité 7 . Leur collecte est affermée à des particuliers qui versent ensuite une somme globale et périodique à la ville, en trois ou quatre «cartons de gabelle» annuels identiques.

L'argent peut aussi provenir d'un emprunt à plus ou moins court terme afin de pallier les problèmes de trésorerie et de s'assurer rapidement de liquidités importantes. Ainsi, en 1498 toujours, la ville d'Avignon emprunte à Pons Lartessuti la somme de deux mille écus d'or au taux de $7 \%$ pour financer les festivités de l'entrée de César Borgia et le présent qui doit lui être offert. Cette décision du Conseil de ville, datée du 5 octobre 1498, intervient après une autre, datée du 2 octobre, indiquant le souhait de la municipalité de lever des pensions sur l'hôtel de ville auprès des citoyens de la ville à hauteur de deux mille écus d'or. Il est donc probable que la municipalité se soit rapidement ravisée en se rendant compte peut-être que la levée d'une telle somme auprès d'une multitude de citoyens serait trop longue, dans un contexte d'urgence.

7. Pour une étude plus complète sur les modes de financement des spectacles, nous renvoyons à M. BonICEL, «Les modes de financement public des spectacles à Avignon à la fin du Moyen Âge », Studies in Early Modern French, 13, 2010, p. 31-40. 


\section{Les prix}

La notion de budget semble absente de la comptabilité avignonnaise. On se demande alors comment les administrateurs municipaux sont capables d'estimer précisément le coût des spectacles qu'ils organisent et la compatibilité de celui-ci avec les sommes qui sont à leur disposition. Si nous n'avons pas de réponse catégorique à cette question, il est en revanche possible d'avancer le fait que leur contact matériel avec l'argent, et une assez grande stabilité des prix de ce type d'activités à la fin du Moyen Âge, leur permettent d'avoir une connaissance intrinsèque des prix habituellement pratiqués et du coût moyen des événements.

Cette connaissance des prix semble transparaître dans un certain nombre de mandats où l'on remarque que le notaire est secrétaire de la ville, lorsqu'il appose l'ordre de paiement sur un mandat qui a été préparé par le créancier luimême avec la liste des prix à payer, raye certains prix pour en noter un autre moins important, parfois précédé de la mention: «réduit à». Il est possible de penser que la municipalité est alors autorisée à fixer elle-même les prix qu'elle souhaite payer en fonction de la connaissance qu'elle a de ceux-ci, ou bien que les prix avaient fait l'objet d'une négociation préalable dont nous n'aurions pas gardé la trace. Dans cette seconde hypothèse, le notaire et secrétaire, au moment de la réception du mandat, serait chargé de faire respecter cet accord.

Si on parvient à connaître le prix de certains éléments comme le textile, les cierges, la fabrication de décors peints par exemple, d'autres sont beaucoup plus délicats à évaluer, notamment ceux des prestations artistiques. En effet, comme nous l'avons dit plus haut, la composition et l'exécution d'un jeu ou d'une danse sont souvent confiées à un artiste, à charge pour lui de recruter et de rémunérer ses compagnons. La part de ce qui revient à l'auteur/meneur de jeu et celle de chacun des prestataires sont alors difficiles à déterminer. Pour les musiciens en revanche, la tâche est plus aisée dans la mesure où certains sont des salariés permanents de la ville (comme les joueurs de trompette) et où d'autres (comme les ménétriers de bas instruments) sont employés directement et font l'objet d'une ligne de paiement particulière dans la comptabilité.

\section{Lieux de spectacles et typologie des performances}

L'étude globale d'un espace géographique et institutionnel précis permet également de repérer un certain nombre de constantes en ce qui concerne l'environnement matériel et géographique des performances. Ainsi, à Avignon, on peut déterminer quels sont les lieux de spectacles le plus souvent utilisés dans la ville (la Maison de la Ville, le Puits-aux-Bœufs, la Porte Saint-Lazare, l'église des Cordeliers...). 
Enfin, l'étude globale du phénomène performatif pour une période et une aire géographique données permet d'établir une typologie de différents événements présentant des caractéristiques communes, par la trace qu'ils laissent dans la documentation. Ainsi, on se rend compte que les entrées solennelles, les banquets de Carême Entrant mais aussi les messes célébrées à l'occasion de funérailles, réelles ou symboliques, de grands dignitaires, sont organisés par le même personnel municipal, mobilisent le même type de fournisseurs et d'artisans, et nécessitent très souvent des volumes financiers comparables.

Cette proximité d'un grand nombre d'événements dans les traces qu'ils nous laissent nous conduit à penser que la société urbaine de la fin du Moyen Âge accorde au fait performatif une place essentielle. De fait, à travers l'étude de ces documents comptables, essentiels à la compréhension de l'organisation de la société urbaine à la fin du Moyen Âge, c'est bien l'omniprésence du fait théâtral dans l'espace public que l'on peut lire en plein ou en creux, selon ce que les mentions disent ou ce qu'il faut en reconstruire en les croisant avec d'autres sources d'archives. Pour souligner et développer ce dernier point, nous proposons à présent de nous pencher également sur un objet plus difficile à discerner immédiatement dans ces sources, mais essentiel à la compréhension du phénomène performatif.

\section{Le public des performances et ses réactions : sources et enjeux historiques}

Parmi les nombreux domaines de recherche des historiens du théâtre médiéval qui restent à (ré-)explorer, l'étude du public des représentations présente en effet un intérêt certain et des difficultés particulières. Intérêt du sujet pour l'histoire des mentalités et pour l'histoire politique et religieuse, si l'on admet que par ce biais on peut éclairer d'un jour nouveau la réflexion sur la formation de l'opinion publique à la fin du Moyen Âge. Difficultés liées à la rareté des sources qui permettraient de documenter la composition de ce public ainsi que sa réaction lors de représentations. Ce dernier point, en particulier, est problématique puisqu'il échappe en partie à la consignation par l'écrit. Cependant, son étude demeure essentielle, car elle permet de déterminer la réception d'une performance par son public, et non pas seulement la réception d'un texte théâtral par un lectorat. Mis en présence d'un enregistrement de ce texte nécessairement partiel sinon partial ${ }^{8}$,

8. De nombreuses questions sont à prendre en compte dans l'analyse d'un support et d'un texte théâtral pour tenter de déterminer son statut par rapport à la performance, notamment celle de savoir à quel moment et pour quel usage ou dans quel but le texte a été mis par écrit, et quel est l'écart entre cet état écrit et celui du texte joué. Ces questions sont déterminantes pour l'étude de la représentation avérée ou possible d'un texte conservé. 
voire confronté à son absence (quand une représentation n'est connue que par des sources d'archives), l'historien peut-il espérer saisir le moment même de la représentation et son impact sur le public de l'époque? En d'autres termes, est-il possible de déterminer quel est (ou quels sont) le(s) public(s) d'une représentation ainsi que l'effet réel d'une performance sur les spectateurs médiévaux ? Cette double question, les historiens du théâtre français ont souvent tenté d'y répondre en étudiant les textes des pièces ${ }^{9}$. Nous ne récusons pas cette approche, qui permet notamment de mettre en lumière le système de communication entre un auteur et son public/lectorat, mais il nous paraît essentiel, en parallèle à l'analyse littéraire des textes théâtraux, d'insister sur la nécessité de prendre en compte les sources historiques, pour montrer en quoi l'étude du public peut nous aider à évaluer l'importance du théâtre comme phénomène social ${ }^{10}$.

\section{L'étude des sources}

Nous laisserons ici de côté la question de la composition du public, dont l'examen dans les sources d'archives peut être effectué selon la méthode proposée plus haut. Une étude attentive des sources comptables publiques et privées peut en effet permettre d'apporter un nombre certain d'informations précises. Pour ne citer que quelques exemples, on peut ainsi, dans un certain nombre de cas, déduire de mentions faites dans les livres de comptes municipaux et ecclésiastiques (comptes de chapitres notamment) si une représentation était publique, et où elle a pu avoir lieu dans la ville (ou hors les murs), et se faire ainsi une idée des conditions dans lesquelles un public choisi (échevins ou personnages importants) pouvait assister à la représentation (emplacement - balcon ou

9. Pour ne citer que quelques exemples d'articles dont le titre mentionne explicitement le public, A. TIssIER considère ainsi en quelques lignes les «procès-verbaux» de représentations de farces et l'iconographie, pour conclure que «les textes des farces sont plus riches de renseignements», dans son article «Le public des farces en France à la fin du Xv siècle et au début du Xvi ${ }^{e}$ siècle», dans $\mathrm{H}$. Kindermann éd., Das Theater und sein Publikum, Vienne, 1977, p. 148-160 (p. 150). J.-Cl. Aubailly ne procède pas autrement dans son article «Le théâtre profane et son public: étude des envois», Tréteaux, 2, 1980, p. 1-15, en cherchant à déterminer quel espace scénique et quel type de public les salutations terminant les farces et sotties permettent de reconstruire; CL. THIRY mène une analyse similaire pour déterminer l'impact du comique des farces sur les spectateurs dans «L'altérité du rire médiéval. La farce et son public», dans G. Smolka-Koerdt, P. Spangenberg et D. Tillmann-Bartylla éds., Der Ursprung von Literatur: Medien, Rollen, Kommunikations-situationen zwischen 1450 und 1650, Munich, 1988, p. 199-221.

10. A.E. KNIGHT a d'ailleurs montré, avec son étude du théâtre des processions lilloises, que l'on peut lier les deux types d'analyse, pourvu que les données d'archives soient véritablement prises en compte. Parmi ses nombreux articles sur le cas lillois, voir à titre d'exemple «Beyond Misrule: Theatre and Socialization of Youth in Lille», Research Opportunities in Renaissance Drama, 35, 1996, p. $73-84$. 
fenêtres d'un bâtiment public ou privé - ou frais de bouche par exemple). On peut encore penser aux tentatives d'évaluation du nombre de spectateurs présents lors d'une représentation payante, à partir d'un bilan financier ${ }^{11}$.

En revanche, nous souhaitons aborder plus longuement la question, à nos yeux problématique pour l'enquête historique, de la description des réactions des spectateurs. Sur le plan méthodologique, il faut en effet être conscient que la description d'une représentation dramatique sert toujours, dans les documents, un but extérieur à la simple description du phénomène performatif. On doit donc toujours lire cette vision à l'aune de l'arrière-plan contextuel, voire idéologique ou symbolique des sources prises en compte. Ceci est particulièrement clair avec les sources narratives (récits d'entrées, chroniques et journaux personnels) qui relatent des représentations dramatiques' Il nous apparaît que c'est moins la description de l'activité théâtrale et son impact sur les spectateurs qui y sont visés, que la portée symbolique de la performance en tant que composante d'une sociabilité urbaine - ou la valeur du message diffusé par les tableaux vivants, dans le cas spécifique des entrées joyeuses ${ }^{12}$. En effet, pour ces dernières, la vision des activités dramatiques donnée souligne essentiellement la stratégie de communication entre les villes et le prince. Le public (quand il est évoqué) y est toujours unanimement appréciateur du spectacle et admiratif du prince et de sa cour, sous la plume de l'indiciaire observant la cérémonie de la perspective du prince comme sous celle du clerc au service de la municipalité, qui veut mettre en avant les bonnes relations entre la ville et le prince.

Il est pourtant une catégorie de sources historiques pour laquelle la réaction du public est essentielle afin de comprendre le phénomène performatif. Les documents de nature juridique - récits de témoins recueillis dans le cadre d'enquêtes sur des représentations ayant causé des troubles de l'ordre public et les réglementations locales décrivant des comportements problématiques du public - permettent en effet de nuancer la vision par trop incomplète d'un public muet ou pacifique lors des représentations. Des témoignages issus des villes du sud des Pays-Bas soulignent ainsi une attitude particulièrement critique d'un public qui utilise l'occasion de se réunir fournie par la représentation comme un espace pour débattre des idées de la Réforme à un moment où les autorités catholiques répriment sévèrement ce type de discussions sur la voie publique. Citons, à titre d'exemple, la représentation en 1563 dans la petite ville de Mouvaux (près de

11. Voir pour l'exemple de la Passion jouée à Valenciennes en 1547, les calculs de L. Petit DE Julleville, Histoire du théâtre en France. Les mystères, Paris, 1880, t. II, p. 152-153, et la réévaluation d'É. Konigson, La Représentation d'un mystère de la Passion à Valenciennes en 1547, Paris, 1969, p. 21-22.

12. Sur les sources et la dimension symbolique de ces spectacles dans l'espace flamand, voir É. Lecuppre-Desjardin, La Ville des cérémonies. Essai sur la communication politique dans les anciens Pays-Bas bourguignons, Turnhout (Studies in European Urban History, 4), 2004. 
Lille) de La Vérité cachée, pièce d'inspiration protestante imprimée par Pierre de Vingles à Neuchâtel en $1533^{13}$. La description de la représentation et des réactions pour le moins critiques du public envers le clergé catholique subsiste dans les témoignages enregistrés lors de l'enquête visant à déterminer qui sont les responsables qui contreviennent à l'ordonnance impériale de 1559 interdisant de mettre en scène des pièces portant sur des sujets religieux sans autorisation préalable. Soulignons ici que, comme pour les autres sources analysées précédemment, il faut considérer avec précaution les témoignages recueillis en posant la question des motivations des déposants, dont on peut se demander en quelle qualité ils sont interrogés: s'agit-il de simples passants ou de représentants des autorités ecclésiastiques et civiles dont l'autorité a été bafouée (comme c'est le cas à Mouvaux) ? Quel intérêt pouvaient-ils avoir à faire une description détaillée des réactions critiques du public?

Une prise en compte des législations locales peut permettre de compléter ces témoignages. Ainsi, une image d'un public actif se dessine, comme à Tournai où des ordonnances locales issues du registre des délibérations échevinales de Tournai faites en 1550 et 1551 soulignent une tendance des spectateurs à se manifester bruyamment dans la même auberge où, neuf ans plus tard, des représentations d'histoires bibliques provoquent dans l'auditoire des réactions de prise de parti nettes sur le plan des idées religieuses ${ }^{14}$.

\section{Spectateurs et opinion publique}

Ces documents, en offrant de précieux renseignements sur les réactions du public à une représentation dramatique, permettent de souligner l'intérêt de prendre en compte le théâtre comme élément de communication et d'expression politique et religieuse à la fin du Moyen Âge et au début de l'époque moderne. Il nous semble en effet, et nous nous inscrivons en cela dans la continuité des recherches les plus récentes sur la question, que le théâtre joue un rôle dans la formation de l'opinion publique pour la période que nous étudions ${ }^{15}$. Pour reprendre l'exemple

13. K. LAvÉANT, «Le théâtre du Nord et la Réforme: un procès d'acteurs dans la région de Lille en 1563 », European Medieval Drama, 11, 2007, p. 59-77, et A. Lotтin, «Un précieux témoignage de la diffusion du calvinisme dans la châtellenie de Lille: La Farce de Mouvaux (1563)», Annales du comité flamand de France, 61, 2003, p. 155-161.

14. Sur les représentations problématiques à Tournai, voir K. LAVÉANT, «Le théâtre et la Réforme dans le nord de la France», dans M. BouhaïK-Gironès, J. Koopmans et K. LAVÉAnT éds., Le Théâtre polémique français (1450-1550), Rennes, 2008, p. 161-177. Les ordonnances sont reproduites par A. Delangre, Le Théâtre et l'art dramatique à Tournai, Tournai, 1905, p. 9.

15. Nous nous inscrivons ici dans la continuité des travaux récents proposés par les historiens médiévistes sur la réévaluation des théories de J. Habermas concernant la notion d'espace public au Moyen Âge. Nous renvoyons en particulier à la très riche analyse de l'impact des textes littéraires 
de Mouvaux, on peut poser l'hypothèse que c'est bien l'ensemble du village qui assiste à la représentation, et qui discute, pendant la représentation, des débats religieux qui agitent la région dans les années 1560, témoignant ici d'un débat public à l'échelle locale ${ }^{16}$. En ce sens, on peut bien parler d'un mouvement de communication réciproque entre la scène et la salle. En effet, la parole théâtrale libère celle du public, qui s'exprime et débat des affaires du temps avant, pendant et après la représentation, profitant de l'occasion fournie par cette dernière pour s'assembler (alors que les rassemblements publics sont étroitement surveillés par les autorités). La mise en scène de questions théologiques couvertes par le jeu des allégories permet ainsi la discussion ouvertement critique au sein du public, et elle témoigne parallèlement du désir de ce public de porter le débat sur la place publique (au sens figuré et au sens propre, grâce au dispositif scénique construit sur la place du village ou improvisé à l'intérieur de l'auberge, autre lieu de sociabilité par excellence). Il ne faut en effet pas oublier que ce sont des habitants du village (dont certains sont fils d'un représentant de l'ordre, qui plus est) qui organisent cette représentation à Mouvaux, bravant sciemment les placards impériaux interdisant de tels événements. Il nous semble que la spécificité du théâtre dans ce cas est de créer, le temps de la représentation, un espace symbolique de discussion et de réflexion, qui échappe au contrôle des autorités par la censure habituelle des textes et de la parole.

De plus, il faut souligner que la représentation théâtrale précède les manifestations plus violentes de contestation religieuse telles qu'elles se produisent en 1566 pendant la vague d'iconoclasme qui touche une grande partie des villes de la région ${ }^{17}$. Il n'est en effet certainement pas anodin que les pièces jouées à Tournai

et en particulier des représentations à caractère dramatique sur la formation de l'opinion publique et son expression dans les Pays-Bas, proposée par J. Bloemendal et A. van Dixhoorn, «The Sharpness of a Honed Tongue. Literary Texts and Public Opinion in the Early Modern Netherlands », dans J. Bloemendal et A. van Dixhoorn éds., Literary Culture and Public Opinion in the Early Modern Low Countries, Leyde, (à paraître).

16. Si l'on en croit les témoins, le public s'élèverait à 1500 spectateurs. En 1505, on compte 104 feux soit environ 520 personnes à Mouvaux. Si l'on table sur une croissance explosive de la population en cinquante ans avec un doublement du nombre de feux similaire à celui de la ville voisine de Tourcoing sur la même période, on obtient un passage à environ 1000 habitants vers 1550 . Même en admettant la présence de spectateurs venus de l'extérieur de Mouvaux et une exagération possible des témoins concernant le chiffre de 1500 spectateurs, on peut envisager sans problème que ce soit bien la quasi-totalité des habitants du lieu qui assistent à la représentation. Pour les chiffres concernant la croissance démographique dans la région, voir A. LotTin et Ph. Guignet dir., Histoire des provinces françaises du Nord, t. 3: De Charles Quint à la Révolution française (15001789), Arras, 2006, p. 29-30, A. LotTin dir., Histoire de Tourcoing, Westhoek, p. 49-55 et p. 62-66, et A. Derville, Enquêtes fiscales de la Flandre wallonne 1449-1549, t. 3 : Les enquêtes de 1498 et 1505, Lille, 2003, p. 177.

17. Mouvaux et Tournai sont également touchées: voir la carte proposée par A. LotTin et S. 
et Mouvaux que nous avons étudiées mettent notamment en scène le prophète Élie vilipendant les statues du dieu Baal et l'épisode célèbre du Veau d'or. On peut alors soumettre l'idée selon laquelle ces représentations ont préparé le terrain pour des actions prolongeant un mouvement d'opinion publique dans ces villes, au moins autant (sinon plus, vu la possibilité de toucher un auditoire important par la représentation en public) que la dispersion de textes copiés ou imprimés ou d'autres modes de propagation des idées de la Réforme.

Ces réflexions, qu'il faudra confirmer par de plus amples recherches dans les archives de la région, illustrent la nécessité de rendre au théâtre la place qu'il mérite comme phénomène de communication sociale et politique de grande ampleur dans les villes de la fin du Moyen Âge.

À travers ces quelques exemples tirés de deux régions, nous espérons avoir montré la nécessité d'un retour aux sources historiques afin de mieux comprendre la place du théâtre dans la société de la fin du Moyen Âge, non plus seulement comme phénomène littéraire, mais également comme vecteur des idées voire des débats qui peuvent occuper l'espace public à cette période. Du fait du volume imparti, nous nous sommes limités à la présentation détaillée d'un type de sources: les documents comptables, et d'un champ d'interrogation: le public des représentations dramatiques tel qu'on peut le saisir, à travers les sources judiciaires, dans son rapport problématique aux idées politiques et religieuses présentées sur la scène.

Le lecteur aura compris que de tels champs d'enquête se multiplient si l'on veut saisir l'ensemble des questions matérielles touchant à la mise en place pratique du cadre de la représentation (scène, espace de jeu dans la ville, autorisation, organisation, financement et direction de la représentation par des «meneurs de jeu»...) aussi bien qu'à l'impact symbolique du phénomène performatif dans l'espace urbain francophone des $\mathrm{XV}^{\mathrm{e}}$ et $\mathrm{XVI}^{\mathrm{e}}$ siècles.

Ajoutons à cela la nécessité de mettre en parallèle des études sur des espaces géopolitiques variés bien que comparables sur le plan linguistique, et le lecteur pourra se faire une idée de l'ampleur de la tâche qui attend les historiens du théâtre dans les décennies à venir - un travail de longue haleine, qui non seulement permettra une meilleure (re-) connaissance du théâtre médiéval mais qui contribuera aussi à un nouveau regard sur la société qui en a fait un élément essentiel de son fonctionnement. 
Matthieu BonICEL - Bibliothèque nationale de France, département des Manuscrits, 58 rue de Richelieu, 75084 Paris

Katell LAVÉANT - Universiteit van Amsterdam - Spuistraat 134, 1012 VB Amsterdam, Pays-Bas

\section{Le théâtre dans la ville: pour une histoire sociale des représentations dramatiques}

Pour évaluer à sa juste mesure la place et l'influence du théâtre comme phénomène social à la fin du Moyen Âge, il faut opérer un retour aux sources historiques, encore trop rarement étudiées pour cerner les cultures dramatiques. Quelles sources sont disponibles pour contribuer à l'histoire du théâtre, et comment les appréhender? Nous proposons ici deux cas d'étude. D'abord, l'étude ciblée d'une zone géographique et de ses archives comptables, à travers l'exemple d'Avignon pour la période 1450-1550, permet de mettre en avant la matérialité de la représentation et l'importance du théâtre comme phénomène social dans l'espace municipal. Puis l'étude de la réception d'une représentation théâtrale par son public, grâce à l'analyse d'archives judiciaires dans les provinces du Nord, pose la question du rôle du théâtre dans la formation d'une opinion publique pendant la Réforme. On peut ainsi saisir l'ensemble des questions matérielles touchant à la mise en place pratique du cadre de la représentation aussi bien qu'à l'impact symbolique du phénomène performatif dans l'espace urbain francophone des $\mathrm{XV}^{\mathrm{e}}$ et $\mathrm{XVI}$ siècles, pour aboutir à une meilleure reconnaissance du théâtre médiéval et un nouveau regard sur la société qui en a fait un élément essentiel de son fonctionnement.

théâtre - comptabilité municipale - archives - justice - cérémonies publiques Provence - Avignon - Nord de la France - Pays-Bas

\section{Drama in the City : toward a Social History of Theatrical Performances}

In order to evaluate the role and influence of drama as a social phenomenon in the Late Middle Ages, one has to go back to the historical sources, that are still rarely studied to understand the theatrical cultures of the time. Which sources are available for the historian of drama, and how can we use them? We propose here two study cases. First, the study of a specific geographical area and its accounting records, with the example of the city of Avignon between 1450 and 1550 , allows understanding the material aspects of the performance as well as the importance of drama in the municipal environment. Then, the study of the reception of a theatrical performance by its audience, thanks to judicial archives in the Southern Low Countries, shows the role of drama in the process of for- 
mation of a public opinion during the Reformation. One can thus comprehend all the material questions linked to organization of a performance as well as the symbolic impact of the performance in the francophone urban environment of the 15th and 16th centuries, in order to shed new lights of medieval drama as well as on the society that used it as an essential element of its functioning.

drama - municipal accounts - archives - justice - public ceremonies - Provence - Avignon - North of France - Low Countries 
